\title{
Emplois de la préposition pour suivie d'une infinitive : description, critères formels et annotation en corpus.
}

\author{
Margot Colinet ${ }^{1}$, Laurence Danlos ${ }^{2}$, Mathilde Dargnat $^{3}$, Grégoire Winterstein ${ }^{4}$ \\ 1. Alpage \& LLF, CNRS \& INRIA Paris-Rocquencourt \& Université Paris Diderot \\ 2. Alpage \& IUF, INRIA Paris-Rocquencourt \& Université Paris Diderot \\ 3. ATILF, CNRS \& Université de Lorraine \\ 4. LPL, CNRS \& Université d'Aix Marseille, et HSS, Nanyang Technological University \\ Margot.Colinet@gmail.com, Laurence.Danlos@linguist.univ-paris-diderot.fr, Mathilde.Dargnat@atilf.fr, \\ Gregoire.Winterstein@gmail.com
}

\section{Introduction}

Cet article sur la préposition pour s'inscrit dans le cadre du projet FDTB (French Discourse Treebank, (Danlos et al., 2012)) dont l'objectif est l'annotation discursive du corpus FTB (French Tree Bank, (Abeillé et al., 2003)) déjà annoté pour la syntaxe. Le projet FDTB se place dans la lignée du projet PDTB (Penn Discourse Treebank, (Prasad et al., 2008)) qui porte sur l'anglais. Une première étape du FDTB consiste à repérer toutes les occurrences de connecteur de discours. Cette étape demande en particulier de déterminer pour chaque occurrence d'un item qui peut être connecteur si elle est effectivement employée comme connecteur. Ainsi et est connecteur en (1a) — marquant un relation de continuation — mais ne l'est pas en (1b).

(1) a. Luc a passé l'aspirateur et il a fait la vaisselle.

b. Luc et Marie se sont mariés en Juin 2009.

Parmi les items qui peuvent être ou non employés comme connecteur, notre attention s'est portée sur la préposition pour suivie d'une infinitive, séquence notée pourVinf. Cette préposition introduit un argument sous-catégorisé (régi) par l'adjectif idéal en (2a) tandis qu'elle fonctionne comme connecteur marquant une relation de discours de but en $(2 \mathrm{~b})$. Autrement dit, le rôle final de pourVinf se calcule au niveau «grammatical» pour (2a) (niveau syntaxico-sémantique qui s'appuie sur un lexique enregistrant les cadres de souscatégorisation des éléments prédicatifs), et au niveau discursif pour (2b) (niveau à l'interface sémanticopragmatique qui intervient après le niveau syntaxico-sémantique).

(2) a. Ce couteau est idéal pour peler les tomates.

b. Luc a fait une pizza pour faire plaisir à Marie.

L'outil Tregex (Levy \& Andrew, 2006) a permis d'extraire du FTB 1162 occurrences de séquences pourVinf. Une première annotation manuelle d'une centaine de ces occurrences réalisée par les auteurs de cet article a vite montré que pour ne fonctionnait pas comme connecteur dans un pourcentage important de cas et qu'il était nécessaire de mettre au point un ensemble de critères formels permettant de distinguer les différents emplois de pour suivie d'une infinitive. Ceci nous a naturellement amenés à étudier la littérature. D'abord les nombreux travaux de Pierre Cadiot sur cette préposition (Cadiot, 1990b ; Cadiot, 1990a) : ces travaux nous ont inspirés mais ne permettent pas directement de définir des critères formels. Ensuite, la grammaire 
de (Wyler, 2013) qui fourmille d'exemples. Enfin, les travaux de lexicographie, par exemple pour les verbes et leurs arguments, les tables du lexique-grammaire (Gross, 1975 ; Tolone, 2013), le lexique des verbes du français (Dubois \& Dubois-Charlier, 1997) et Dicovalence (Van den Eynde \& Mertens, 2003) ${ }^{1}$. Il nous est vite apparu que ces ressources lexicographiques avaient passé sous silence de nombreux cas où la préposition pour est sous-catégorisée par un verbe : ainsi même si pour commute avec à en (3), seule la préposition à est répertoriée dans ces lexiques qui font référence dans la communauté francophone.

(3) Luc a entraîné son chien à/pour guider les aveugles.

Cet article se donne trois objectifs :

- dresser la liste des différents emplois de la préposition pour introduisant une infinitive, en précisant ses relations par rapport au reste de la phrase, Section 2 ;

- établir, si nécessaire, des critères formels permettant de séparer ces différents emplois, Sections 3 et 4 ;

- donner le bilan d'une annotation des pourVinf du FDTB, Section 5.

\section{Les différents emplois de pour introduisant une infinitive}

Cette section se contente de lister les différents emplois de pour introduisant une infinitive, les critères permettant de différencier ces emplois étant présentés dans les deux sections suivantes, qui préciseront des notions comme pour connecteur versus pour sous-catégorisé. Les exemples présentés sont soit issus de corpus (du FTB — ils sont alors suivis d'un numéro — ou d'un autre corpus) soit construits ; les séquences pourVinf sont mises en italiques.

\subsection{Pour est connecteur de discours}

Lorsque la préposition pour joue le rôle de connecteur de discours, la séquence pourVinf est qualifiée de "subordonnée adverbiale (infinitive)". Le connecteur pour sert la plupart du temps à marquer une relation de discours finale, causale ou temporelle. Dans le cas d'une relation finale, la subordonnée indique le but du procès décrit dans la matrice, (4); pour commute alors avec afin de ou dans le but de.

(4) Luc a fait une pizza pour faire plaisir à Marie.

Dans le cas d'une relation causale, la subordonnée indique la cause du procès décrit dans la matrice, (5); pourVinf peut alors être remplacée par une subordonnée à temps fini introduite par parce que.

(5) L' an dernier, le correspondant du quotidien britannique Financial Times s'est fait expulser pour avoir fait état de "l'évaporation" des énormes bénéfices tirés des exportations de pétrole pendant la guerre du Golfe. (4701)

Pour les relations temporelles, on peut distinguer :

- une relation de succession où la subordonnée indique la suite du procès décrit dans la matrice, (6a); pour commute alors avec avant de;

- une relation de succession où la subordonnée indique la suite et le résultat du procès décrit dans la matrice, (6b); 
- une relation de concomitance, (6c-e); pourVinf peut alors être remplacée par une subordonnée à temps fini introduite par lorsque.

(6) a. Paul est sorti pour revenir aussitôt. (Cadiot, 1990a)

b. De son côté, la construction de logements reprend effectivement, après une forte baisse en 1991, pour remonter à un rythme annuel de 1,3 million de mises en chantier contre 1 million l'année précédente...(2754)

c. Il gémit pour descendre de son lit. (Montherlant) (Wyler, 2013)

d. Sa main tremblait pour écrire. (Cadiot, 1990a)

e. Ils ont, pour quitter la terre natale, mis leurs meilleurs vêtements. (Duhamel) (Wyler, 2013)

Le connecteur pour peut aussi, dans des cas rarement rencontrés dans le FTB, marquer une concession, (7a) ou une « cause pragmatique », $(7 \mathrm{~b})^{2}$.

(7) a. Pour être informaticienne, elle n'est pas moins sexy. (Wyler, 2013)

b. Mais qu'ai-je fait au Bon Dieu pour avoir une femme qui boit? (Jean Saint-Hamond)

\subsection{Pour introduit un complément sous-catégorisé}

La préposition pour peut introduire un complément sous-catégorisé (régi) par un prédicat. Ce prédicat, souligné dans les exemples linguistiques, peut être de trois types :

- un prédicat verbal, qui peut être un verbe simple, (8a), ou une expression verbale composée, (8b),

- un prédicat adjectival, qui peut être un adjectif, (8c), ou un participe passé, (8d),

- un prédicat nominal, qui peut être la tête d'un GN, (8e), ou d'une construction à verbe support, (8f).

(8) a. Luc a utilisé de l'alcool pour enlever son papier peint.

b. La machine de propagande soviétique est déjà à l'oeuvre en dehors de tout concours allemand, pour promouvoir la neutralisation de l' Allemagne. (14065)

c. Idéal pour regarder la télé, « Clipper 5 » coûte 18000 francs. (16184)

d. Un musée conçu pour accueillir des formes d'art très variées. (Le Monde Culture et Idées)

e. Cet ancien élève de l'INSEAD, qui a travaillé aux Etats Unis, ajoute à ses talents de gestionnaire, un sens inné du contact humain et une imagination sans limites pour motiver ses troupes. (5368)

f. Cet ancien élève de l'INSEAD a une imagination sans limites pour motiver ses troupes.

A ces trois types, nous ajoutons le type adverbial pour rendre compte d'exemples comme (9) où pourVinf est appelé par un adverbe d'intensité comme trop ou insuffisamment.

(9) a. Luc est trop bête pour comprendre.

b. Elle est bien trop mignonne pour y toucher ! (Cadiot, 1990a)

c. Le tourisme sert trop souvent de premier emploi, de petit job d'été, de point de passage à l'entrée dans la vie active à des jeunes insuffisamment formés pour pouvoir y envisager une véritable carrière. (5308) 


\subsection{Pour introduit une "relative sans mot QU"}

Une séquence pourVinf peut modifier un nom concret comme dans le GN sujet de (10a). Ce modifieur nominal, sémantiquement équivalent à conçu/prévu/fait pourVinf, (10b), est qualifié de «relative sans mot QU» en adaptant le terme de (Huddleston \& Pullum, 2002) ${ }^{3}$.

(10) a. Un pont pour franchir l'Amazone a été construit en 1745.

b. Un pont conçu/fait pour franchir l'Amazone a été construit en 1745.

\subsection{Pour introduit une expression métadiscursive}

Les pourVinf métadiscursives sont des expressions relativement figées, souvent incidentes, (11). Elles servent à situer un acte de langage par rapport à certaines attitudes du locuteur (sincérité, contrôle de l'économie du discours). Elles sont parfois classées comme des adverbiaux au niveau énonciatif, ou comme des connecteurs (Rossari, a paraitre) et, à ce titre, relèvent nettement des «marqueurs pragmatiques ».

(11) a. Des firmes comme Elf-Aquitaine, Alcatel-Alsthom, Total, PSA Peugeot, Renault, RhônePoulenc, Usinor- Sacilor, Pechiney, Michelin, Saint-Gobain ou Thomson, pour ne citer qu'elles, exportent plus de la moitié de leur chiffre d'affaires. (3448)

b. Cette semaine encore, et pour conclure, Hachette s'est un peu repris. (844)

c. Pour le dire autrement : vous voulez supprimer le chômage ? (1951)

\subsection{Bilan}

Nous avons donc identifié quatre classes d'emplois de pour introduisant une infinitive, avec éventuellement des sous-classes ${ }^{4}$. Ceci conduit pour l'annotation (Section 5) à un jeu de neuf étiquettes : trois étiquettes où pour est considéré comme connecteur, CONN-But/Cause/Narr, et six étiquettes où pour n'est pas connecteur, CAT-V/N/ADJ/ADV plus RelPour et META.

Certaines de ces classes ne posent aucun problème d'identification. C'est le cas des expressions métadiscursives qui ne nécessitent pas de critères particuliers pour être distinguées des autres. A l'inverse, certaines classes ont des frontières beaucoup plus floues et nécessitent d'expliciter des critères aussi formels que possibles pour les différencier. C'est le cas en particulier des emplois de pour comme connecteur versus pour introduisant un complément sous-catégorisé par un verbe. La limite entre ces deux emplois est d'autant plus ténue que la sémantique des compléments pourVinf sous-catégorisés par un verbe peut être finale, causale ou temporelle comme c'est le cas pour la sémantique des subordonnées adverbiales introduites par le connecteur pour (Section 2.1) : les exemples (12a-c) illustrent des complément sous-catégorisés dont la sémantique est respectivement finale, causale et temporelle (rappelons que cette sémantique peut être calculée au niveau grammatical moyennant des lexiques appropriés).

(12) a. Luc a utilisé de l'alcool pour enlever son papier peint. ${ }^{5}$

b. Luc a récompensé Max pour avoir fait la vaisselle.

c. La maîtresse a attendu la fin de la récré pour engueuler Max. 
De plus, une même phrase matrice peut être prolongée par un complément sous-catégorisé ou par une subordonnée adverbiale, $(13 a-b)^{6}$. Il faut donc prendre en compte le contenu sémantique de l'infinitive pour déterminer la nature de son rattachement à la matrice sans se reposer aveuglément sur le prédicat verbal de la matrice (ce qui est source d'erreur dans l'annotation, Section 5).

(13) a. Luc traîne pour/à nous répondre.

b. Luc traîne pour/*à nous répondre le plus tard possible.

Face à ces difficultés, nous consacrons la section suivante à définir des critères permettant de distinguer les emplois de pour comme connecteur de ceux où il est introducteur de compléments sous-catégorisés par un verbe (ou une expression verbale composée).

Les cas d'emploi de pour introduisant un complément sous-catégorisé par un adjectif ou un adverbe ne posant pas de problème d'ambiguïté, nous ne leur consacrons pas de section particulière. Par contre, les cas d'emploi de pour introduisant un complément sous-catégorisé par un nom pouvant être confondus avec les cas de relatives sans mot QU- modifiant un nom, nous dédions la Section 4 à ces deux cas.

\section{Connecteur versus complément sous catégorisé par un verbe}

Discuter de la différence entre les emplois de pour comme connecteur versus introducteur de complément sous-catégorisé par un verbe revient à discuter de ce qui est connu en linguistique comme la différence entre ajout et argument. De nombreux critères ont été avancés dans la littérature, tant en lexicographie (Boons et al., 1976 ; Lamiroy, 1983 ; Guillet \& Leclère, 1992) qu'en syntaxe et sémantique (Milner, 1982 ; Bonami, 1999).

Le critère sémantique le plus utilisé est qu'un argument est sémantiquement obligatoire contrairement à un ajout. Ceci se traduit, pour ce qui nous concerne, par le fait qu'une subordonnée adverbiale pourVinf peut être supprimée sans que cela n'affecte de façon critique l'acceptabilité et le sens de la phrase matrice, (14a-b), tandis que la suppression d'un complément pourVinf peut donner un résultat inacceptable lorsque le complément n'est pas récupérable dans le contexte gauche de la phrase. Ainsi, la phrase (15b) est inacceptable (ininterprétable) si on ne peut pas récupérer l'argument de se dépêcher dans le contexte gauche : le discours (15c) est cohérent, l'argument de se dépêcher étant implicite.

(14) a. Luc a fait une pizza pour faire plaisir à Marie.

b. Luc a fait une pizza.

(15) a. Luc s'est dépêché pour finir sa thèse.

b. Luc s'est dépêché.

c. Luc a fini sa thèse la semaine dernière. Il s'est dépêché car il veut soutenir avant Juin.

Le critère syntaxique le plus utilisé est qu'un ajout est facilement antéposable, contrairement à un argument, (16a-b). Soulignons que ce critère doit s'interpréter comme une contrainte préférentielle et non comme une contrainte forte inviolable. Ainsi une subordonnée adverbiale pourVinf dont la valeur sémantique est une succession temporelle ne peut pas s'antéposer, (16c-d). A l'inverse, dans notre annotation du FTB, nous avons trouvé quelques (rares) exemples d'argument verbal ou nominal antéposé, (16e).

(16) a. Pour faire plaisir à Marie, Luc a fait une pizza. ${ }^{7}$ 
b. ? Pour finir sa thèse, Luc s'est dépêché.

c. Luc a marqué un essai pour le transformer aussitôt.

d. \#Pour le transformer aussitôt, Luc a marqué un essai.

e. Pour contourner cet obstacle juridique, une astuce a été trouvée. (1502)

Le caractère sémantiquement obligatoire d'un argument pouvant être sujet à caution (avec des compléments «plus obligatoires » que d'autres, Section 3.6) et la possibilité d'antéposition étant une préférence plutôt qu'une contrainte forte, nous allons proposer une batterie de critères qui aident à déterminer si pourVinf est un argument ou un ajout.

Auparavant, signalons que les verbes sous-catégorisant un complément pourVinf indiquant la cause du procès décrit dans la matrice sont sémantiquement bien délimités : il s'agit de verbes de punition/récompense/félicitation, (17), qui appartiennent à la Table 13 de (Gross, 1975) où seule la préposition de, qui commute avec pour (Luc a puni Ida de/pour avoir sécher le cours), est codée. Dans le même ordre d'idées, les verbes sous-catégorisant un complément pourVinf à valeur temporelle se limitent au verbe attendre, (18a), si on met de côté les verbes prendre/donner/mettre/disposer de/... suivi d'un objet dénotant une durée (18b). La suite de la discussion ne concerne donc que des tests pour déterminer si une séquence pourVinf à valeur finale est un complément sous-catégorisé par le verbe matrice ou une subordonnée adverbiale introduite par un connecteur.

(17) a. Au cours de cette même audience, le tribunal a aussi condamné deux dirigeants de Docks de France à 50000 francs d'amende pour avoir négligé de déclarer le franchissement de seuil. (2215)

b. Le principal responsable de la première banque d' Arabie Saoudite et un associé ont été inculpés, mercredi 1 juillet à New York, par un grand jury pour avoir escroqué ...(2575)

(18) a. Je trouve scandaleux et anormal que les opérateurs aient attendu la fin de l'année pour prendre une décision brutale de licenciement des deux tiers du personnel sans qu'aucun plan de redressement n'ait été examiné en cours d' année. (988)

b. Luc a mis/pris/s'est donné trois mois pour finir sa thèse.

\subsection{Commutation avec la préposition à, de ou contre}

La préposition pour commute parfois avec une autre préposition introduisant un complément considéré comme sous-catégorisé dans toutes les ressources lexicographiques connues (Gross, 1975 ; Tolone, 2013 ; Dubois \& Dubois-Charlier, 1997) : il est alors naturel de poser que pour est aussi sous-catégorisée. Nous avons identifié les cas suivants :

- pour commute avec de après des verbes comme se dépêcher, se dégrouiller, se hâter, s'empresser, ..., (15a), et, rappelons-le, après des verbes de punition/récompense/félicitation pour introduire un complément exprimant la cause du procès de la matrice;

- pour commute avec à après des verbes comme oeuvrer, lambiner, traîner, ..., (19a), après des verbes comme engager, entrainer, solliciter, ..., qui se construisent avec un objet direct contrôlant l'infinitive, (19b), et après des verbes comme dévouer, gaspiller, utiliser, ..., qui se construisent avec un objet direct introduit par un déterminant possessif référant obligatoirement au sujet, $(19 \mathrm{c})$; 
(19) a. Luc a oeuvré à/pour vous protéger.

b. Luc emploie Max à/pour surveiller Ida.

c. Luc gaspille ses forces à/pour étudier cette seule question.

- pour suivi d'un GN commute avec contre après batailler, combattre, intriguer, manifester, magouiller, plaider, voter, ..., (20a); seule pour peut introduire une infinitive, (20b), cette préposition est alors souscatégorisée comme lorsqu'elle est suivie d'un GN.

(20) a. Luc a bataillé contre/pour cette proposition

b. Luc a bataillé * contre/pour faire passer/bloquer cette proposition.

Enfin, selon (Gross, 1975 ; Lamiroy, 1983), pour commute avec la préposition vide $\varnothing$ après un verbe de mouvement, (21a-b).

(21) a. Luc est allé à la boulangerie $\varnothing /$ pour acheter des croissants.

b. Luc a filé à la gare ø/pour retrouver Ida.

\subsection{Contrôle de l'infinitive}

Lorsque la préposition pour est connecteur, elle est généralement considérée comme une forme "réduite" de la conjonction de subordination pour que : la préposition remplace la conjonction lorsque le sujet de la subordonnée adverbiale est coréférent au sujet de la phrase matrice, (22a-b).

(22) a. Luc s'est levé de bonne heure pour que Marie attrape son train.

b. Luc s'est levé de bonne heure pour attraper son train.

Le sujet de la subordonnée adverbiale pourVinf est donc normalement contrôlé par le sujet de la phrase matrice. Il existe cependant quelques exceptions : dans le fameux exemple (23a), le sujet de la subordonnée n'est pas exprimé explicitement mais on comprend qu'il s'agit des propriétaires de la maison. En (23b-c), le sujet de l'infinitive est coréférent à l'objet de la matrice, l'infinitive étant au passif.

(23) a. ? La maison a brûlé pour toucher l'assurance. (Zubizareta, 1985)

b. On l'avait mise au pensionnat de Châteaudun, pour y être élevée. (Zola) (Wyler, 2013)

c. On le ramènerait en France pour y être jugé. (Wyler, 2013)

En dehors de ces cas assez marginaux, le contrôle de l'infinitive (à l'actif) par l'objet direct de la matrice, (24), ou par l'objet indirect ou un complément de nom de l'objet indirect, (25), constitue un test permettant de dire que pour est sous-catégorisée par le verbe de la matrice.

(24) a. Le gouvernement a réquisitionné les militaires pour évacuer les blessés.

b. Max entraîne son chien à/pour guider les aveugles.

c. Ce succès qualifie cette équipe pour disputer la finale.

d. Luc a condamné Max de/pour avoir refusé cette offre.

(25) a. Nous comptons sur M. Barre pour ne pas provoquer d'incident.

b. Nous faisons confiance au patriotisme de M. Barre pour ne pas provoquer d'incident. (Cadiot, 1990a) 


\subsection{Ajout de la séquence et ce}

Une subordonnée adverbiale pourVinf — et plus généralement un ajout — peut être précédée de la séquence et ce (de préférence suivie d'une virgule) où le pronom ce anaphorise la phrase matrice lorsque celleci n'est pas sous la portée d'une négation ${ }^{8},(26 a-b)$. A l'inverse, cette séquence ne peut pas précéder un argument : le résultat obtenu est mal formé lorsque l'argument est clairement sémantiquement obligatoire car la phrase matrice est alors mal formée, $(27 \mathrm{a}-\mathrm{b})$; le résultat est déviant lorsque l'argument n'est pas clairement obligatoire, $(27 \mathrm{c}-\mathrm{d})$.

(26) a. Luc a fait une pizza et ce, pour faire plaisir à Marie.

b. Face à un besoin croissant de considérations pratiques et éthiques dans la prise en charge des personnes en fin de vie, il paraît primordial de donner un éclairage sur la sédation terminale et ce, pour la différencier de l'acte d'euthanasie. (Google)

(27) a. ? Luc s'est dépêché et ce, pour finir sa thèse.

b. *Il faut être fou et ce, pour aller à Moscou à Noël.

c. ? Luc a utilisé de l'alcool et ce, pour enlever son papier peint.

d. ? Luc est allé à la boulangerie et ce, pour acheter des croissants.

\subsection{Opérateurs sémantiques}

Les possibilités de réfutation diffèrent selon que pour introduit un complément de verbe ou est employé comme connecteur, comme en attestent les interprétations d'une réfutation simple du type C'est faux en réponse à une assertion contenant pourVinf. Lorsque pour introduit un complément de verbe, l'interprétation la plus naturelle d'une réfutation est de porter sur le contenu de la matrice. Ainsi en (28), la réfutation est naturellement interprétée comme Luc ne s'est pas dépêché plutôt que Luc s'est dépêché mais pas pour finir sa thèse.

- Luc s'est dépêché pour finir sa thèse.

- C'est faux.

A l'inverse, lorsque pour est connecteur, la réfutation est ambigüe et (29) peut signifier soit que Luc n'a pas fait de pizza (réfutation du contenu de la matrice) soit que Luc a fait une pizza mais pas pour plaire à Marie (réfutation du but de Luc). En d'autres termes, lorsque pour est un marqueur de relation de discours (finale), le but qu'il introduit est soumis à l'approbation de l'allocutaire, de la même manière que le contenu de la matrice.

\section{- Luc a fait une pizza pour faire plaisir à Marie.}

- C'est faux.

Ces données sont corroborées par des différences d'intonation dans les interrogatives : lorsque pour est connecteur, l'intonation peut porter sur le but, le contenu de la matrice devenant présupposé et passant en arrière plan, (30a). Une intonation équivalente n'est pas possible lorsque pour est sous-catégorisée par le verbe, $(30 b)^{9}$. 
(30) a. Est-ce que Luc a fait une pizza POUR FAIRE PLAISIR À MARIE ? (ou pour autre chose ?)

b. ? Est-ce que Luc s'est dépêché POUR FINIR SA THÈSE? (ou pour autre chose ?)

Indiquons au passage que lorsque la préposition pour est sous-catégorisée, elle peut parfois apparaître seule, c'est-à-dire sans son objet, (31), mais ceci est totalement exclu lorsqu'elle est connecteur.

\section{- Est-ce que cette salle a été prévue pour accueillir 500 personnes?}

- Oui, elle a été prévue pour.

On observe aussi des différences d'interprétation lorsque la phrase matrice comporte une négation. Lorsque pour est connecteur, la négation peut ne porter que sur le but, en particulier lorsque l'énoncé ne peut pas signifier que la phrase matrice est niée comme en (32a) (Amsili, a paraitre). Lorsque pour est souscatégorisée, la négation peut ne porter que sur le contenu de la matrice, (32b), ou porter sur la phrase entière, (32c).

(32) a. Luc n'a pas acheté cette voiture pour s'en séparer.

$\rightsquigarrow$ Si Luc a acheté cette voiture, c'est pas pas pour s'en séparer

b. Deschamps n'a pas magouillé pour prendre la place de Blanc. (Google)

$\rightsquigarrow$ Deschamps a pris la place de Blanc sans magouiller

c. Luc ne passe pas pour avoir du talent.

$\rightsquigarrow$ Il est faux que Luc passe pour avoir du talent

Quelques remarques sur la factivité de pourVinf. Lorsque pour est un connecteur introduisant un but, la phrase matrice décrit une action intentionnelle dont la réalisation augmente la probabilité que le but soit atteint, sans qu'il soit forcément atteint. Citons (Amsili, a paraitre) : « les finales, comme (33a), sont compatibles aussi bien avec une continuation qui implique la non-réalisation de la situation visée, (33b), qu'avec une continuation qui implique sa réalisation, (33c)». Ajoutons que la continuation en (33c) ajoute une information temporelle sur la réalisation de la situation visée (en moins d'un mois) mais qu'un tel ajout d'information n'est pas nécessaire, (33d).

(33) a. Paul a rencontré un médecin pour arrêter de fumer.

b. ... mais il n'a pas réussi à arrêter.

c. ....et il a arrêté en moins d'un mois.

d. ....et il a arrêté.

Lorsque pour sous-catégorise un complément final, la situation visée est souvent réalisée «par défaut »: ainsi une continuation de (34a) qui implique la non réalisation est possible mais difficile, (34b), et une continuation qui implique sa réalisation sans ajout d'information est perçue comme redondante, (34c).

(34) a. Paul a utilisé une perceuse pour accrocher ce tableau.

b. ... ? mais il n'a pas réussi à l'accrocher.

c. ... ? et il a l'a accroché.

Cette factivité par défaut des pourVinf sous-catégorisées est à relier à l'interprétation préférentielle de la réfutation ou de la négation qui ne porte pas sur le but visé. Elle implique de plus que des paires de phrases comme (35-37) — qui sont telles que, dans les exemples en b, un groupe prépositionnel résume le moyen ou la modalité de la matrice en a - peuvent être énoncées dans les mêmes conditions de vérité avec un simple changement de focus/thème. 
(35) a. Paul a utilisé une perceuse pour accrocher ce tableau.

b. Paul a accroché ce tableau avec une perceuse.

(36) a. Paul a magouillé pour obtenir ce poste.

b. Paul a obtenu ce poste par des magouilles.

(37) a. Paul s'est hâté pour finir sa thèse.

b. Paul a fini sa thèse à la hâte.

A rebours, la non-factivité des pourVinf finales où pour est connecteur fait que les conditions de vérité d'exemples comme (38a-b) ne sont pas équivalentes.

(38) a. Paul a fait une pizza pour faire plaisir à Marie.

b. Paul a fait plaisir à Marie avec une pizza.

\subsection{Co-occurrence et coordination de deux séquences pourVinf}

Les phrases matrices sont identiques dans la paire en (39a-b), et d'après les différents critères vus précédemment pour est sous-catégorisée en (39a) et connecteur en (39b) - ce qui montre à nouveau que le contenu de l'infinitive doit être pris en compte pour déterminer le statut de pour. L'acceptabilité de (39c) indique que les deux infinitives introduites par pour peuvent co-occurrer dans la même phrase (sans effet d'itération) et donc qu'elles ne jouent pas le même rôle par rapport à la phrase matrice, ce qui confirme notre position consistant à donner deux statuts différents aux deux occurrences de pour. En (39c), l'ordre des groupes prépositionnels est l'ordre préférentiel, à savoir argument avant ajout, l'ordre inverse débouchant sur un résultat inacceptable, $(39 \mathrm{e})$.

(39) a. Luc a pris un taxi pour aller à l'aéroport.

b. Luc a pris un taxi pour rattraper son retard.

c. Luc a pris un taxi pour aller à l'aéroport pour rattraper son retard.

d. *Luc a pris un taxi pour rattraper son retard pour aller à l'aéroport.

Ces données permettent de définir un nouveau test : est-ce qu'une phrase comportant une infinitive en pour peut être prolongée par une subordonnée adverbiale de but (sans effet d'itération) ? Si oui, l'infinitive ne met pas en jeu une occurrence de pour comme connecteur. Dans le même ordre d'idées, les exemples en (40) mettent en jeu un zeugme dû au fait que les pourVinf coordonnées entretiennent des relations sémantiques différentes avec le contenu de la matrice.

(40) a. A-t-il pris un taxi pour aller à l'aéroport ou pour rattraper son retard?

b. S'est-il endetté pour acheter un appartement ou pour ennuyer son ex-femme? 


\subsection{Bilan}

Les tests que nous avons présentés se veulent aussi opératoires que possible. Il n'empêche qu'ils peuvent être difficiles à évaluer, ce qui pose problème pour l'annotation et l'accord inter-annotateurs (Section 5). Face à de telles difficultés, les linguistes ont souvent recours à des gradiences, verbalisées de la façon suivante : la préposition pour est "plus sous-catégorisée ici que là" ou est "plus connecteur ici que là" (Charolles \& Lamiroy, 2012). Cette position est peut être sage — le futur le dira — mais elle demande un travail d'annotation d'un type particulier : il faut non seulement demander aux annotateurs d'attribuer une note sur une échelle de gradience mais en plus mettre au point des méthodes statistiques non triviales pour évaluer l'accord inter-annotateurs et surtout essayer de tirer des conclusions/généralités à partir des résultats de l'annotation. Nous n'avons pas adopté une telle approche, cherchant à obtenir un jugement binaire : pour est connecteur ou non. Cette attitude est celle adoptée dans tous les travaux d'annotation discursive, dont le PDTB dont nous nous inspirons pour le FDTB. Signalons à ce propos que le PDTB a annoté les cent connecteurs dits les plus fréquents de l'anglais mais pas la préposition to suivie d'une infinitive, qui peut être considérée comme l'équivalent de pour suivie d'une infinitive et qui pose aussi des problèmes fort délicats pour déterminer si l'on est en présence d'un connecteur ou non (Huddleston \& Pullum, 2002, pages 1222-1225).

\section{Compléments sous-catégorisé par un nom et relatives sans mot QU}

\subsection{Compléments sous-catégorisés par un nom}

De même que certains verbes (prédicatifs) sous-catégorisent un complément pourV-inf, certains noms prédicatifs sous-catégorisent de telles séquences : c'est le cas, entre autres, pour les nominalisations des verbes sous-catégorisant un complément en pourVinf ${ }^{10}$, (41), et pour d'autres noms prédicatifs sans relation morphologique avec un verbe, (42).

(41) a. Les magouilles de Luc pour obtenir ce poste sont impressionnantes.

b. La bataille de Luc pour faire passer cette proposition est impressionnante.

c. La tension entre l'Europe et les autres pays participants, notamment les Etats-Unis, à propos de ces négociations commerciales internationales — Uruguay round — , risque de s'envenimer encore avec la condamnation, le 15 janvier, par une instance d'arbitrage du GATT, de Deutsche Airbus — le partenaire allemand du consortium —, pour avoir bénéficié de la part du gouvernement de Bonn d'un système contestable de garantie de change. (1821)

(42) a. Les facultés de Luc pour comprendre les math sont impressionnantes.

b. Malgré le coup de semonce du conflit de Cléon, Renault n'est pas encore parvenu à définir un nouveau calcul de l'intéressement aux résultats et, en dépit de louables efforts pour intégrer de jeunes salariés sans qualification, continue de réduire ses effectifs de plus de 2000 chaque année. (7873)

Un nom prédicatif peut être employé avec un verbe support pour former un prédicat verbal complexe (avoir les facultés pour, faire des efforts/magouilles pour). Lorsqu'un nom prédicatif est employé avec son verbe support, les critères présentés à la section précédente peuvent être utilisés pour montrer que la séquence 
pourVinf est sous catégorisée par le prédicat verbal complexe formé du verbe support et de son nom prédicatif. Ainsi en (43), la réfutation (Section 3.4) est naturellement interprétée comme Luc n'a pas fait de magouilles plutôt que Luc a fait des magouilles mais pas pour obtenir le poste de PI.

\section{- Luc a fait des magouilles pour obtenir le poste de PI.}

- C'est faux.

Il existe d'autres noms sous-catégorisant un complément en pour que ceux mentionnés ci-dessus, à savoir des noms comme astuce, méthode, moyen, procédé, solution, stratégie, technique, truc, ..., (44a). Les verbes supports de tels noms sont des verbes comme trouver, inventer ou créer, (44b).

(44) a. La véritable solution pour dégonfler les sicav monétaires, qui ont fait de la France un "pays de rentiers", serait une baisse spectaculaire des taux à court terme : (11894)

b. Luc a trouvé une astuce pour peler les tomates.

Il est traditionnellement considéré qu'un nom prédicatif réfère à une éventualité (e.g. condamnation) ou une propriété (e.g. facultés). Ce n'est pas le cas des noms de la famille de astuce mais le fait qu'ils souscatégorisent un complément en pourVinf amène à les considérer comme des noms prédicatifs.

\subsection{Relatives sans mot QU}

Nous réservons le terme d'artefact à des objets concrets fabriqués par l'homme ${ }^{11}$. Les noms d'artefact permettent de construire des GN comportant une séquence pourVinf, (45a). Dans (Cadiot, 1990b), il est indiqué que pour dans ces exemples est paraphrasable par destiné $(e)(s)$ à. Nous préférons une paraphrase en conçu/prévu/fait/(e)(s) pour qui permet de garder la préposition pour. Insistons sur le fait qu'une relative sans mot QU fonctionne comme un modifieur (ajout) et non comme un argument. En effet, un nom d'artefact ne peut pas être considéré comme un nom prédicatif dans la mesure où il n'a besoin d'aucun argument syntaxique ni sémantique : il se suffit à lui-même pour introduire un référent. On remarque que le contenu de l'infinitive peut correspondre au rôle télique (Pustejovsky, 1995) du nom d'artefact : ainsi le rôle télique de pont est franchir (un cours d'eau). On peut même observer des exemples où l'infinitive se limite au verbe télique du nom d'artefact, (45b), sans qu'un effet de redondance se fasse ressentir. La préposition pour peut parfois commuter avec $\grave{a}$.

(45) a. Un pont pour franchir l'Amazone a été construit en 1745.

b. Luc a un lit pour dormir.

c. Luc a acheté un couteau à/pour peler les tomates.

Nous avançons qu'un exemple comme (46a) met en jeu une relative sans mot QU. En effet, pour ne peut pas être considérée comme connecteur ni comme sous-catégorisée par théories : l'analyse de pour comme connecteur est éliminée non seulement parce que la suppression de pourVinf rend l'exemple déviant mais aussi par le fait que pourVinf peut être déplacée à l'intérieur du GN sujet sans induire de changement de sens. L'analyse de pourVinf comme sous-catégorisée par théorie(s) ne tient pas en regard du fait que ce nom prédicatif régit un complément en sur, (46b). Au total, il semble licite de considérer que (46a) met en jeu une relative sans mot QU (détachée de son antécédent).

(46) a. De nombreuses théories furent échafaudées pour en rendre compte. (1008) 
b. La théorie de Chomsky sur l'aspect est attendue.

Enfin, on observe des GN comportant une pourVinf dont le nom tête est un nom humain désignant une fonction, par exemple, le titre en (47a) qui est un des 1120000 résultats ramenés par Google pour la requête "soldats pour protéger". Cette séquence s'observe souvent après des verbes comme déployer ou envoyer, (47b-c) et on peut alors envisager qu'elle soit sous-catégorisée par ces verbes. Mais, pour un exemple comme (47d), construit avec le verbe compter d'aspect statique et dont le sujet est non agentif, il semble impossible de considérer que compter sous-catégorise une pourVinf et impossible de poser que pour est un connecteur marquant le but. La solution consistant à poser que pourVinf est une relative sans mot QU semble donc la meilleure.

(47) a. Des soldats pour protéger l'Amazonie

b. Soudan du Sud: les Etats-Unis déploient 45 soldats pour protéger leur personnel.

c. Syrie: La Russie prête à envoyer des soldats pour protéger son personnel sur place.

d. La Colombie ne comptait que 14000 soldats pour protéger un territoire de plus d'un million de Km2.

\subsection{Bilan}

Les GN comportant une séquence pourVinf ont comme nom tête :

- soit un nom prédicatif sous-catégorisant cette séquence : le nom prédicatif désigne une éventualité (bataille, campagne), une propriété (faculté, imagination) ou un nom de la famille de astuce,

- soit un nom d'artefact où cette séquence est un modifieur qualifié de relative sans mot QU,

- soit un nom prédicatif comme théorie, livre, message, conférence, session, ... qui sous-catégorise un argument de «contenu informatif» introduit par la préposition sur mais qui permette de donner des informations sur le contenu informatif via une relative sans mot QU en pourVinf,

- soit un non humain désignant une fonction où pourVinf est une relative sans mot $\mathrm{QU}^{12}$.

Indiquons qu'au cours de l'annotation des pourVinf, il y a eu quelques hésitations entre sous-catégorisation par un nom ou relative sans mot QU, mais que ces hésitations n'ont pas d'incidence pour l'objectif du FDTB, à savoir identifier les connecteurs de discours.

\section{Annotation du FTB}

Dans une première phase, il a été extrait 150 phrases du FTB contenant au moins une infinitive en pour, ce qui fait un total de 156 séquences pourVinf. Deux annotateurs (parmi les auteurs de cet article) ont ensuite annoté chacune de ces 156 occurrences de la préposition pour avec l'étiquette CONN lorsqu'il s'agit d'un connecteur et l'étiquette CAT lorsque la préposition pour introduit un complément sous-catégorisé par un élément de la phrase. Pour chacune des occurrences de pour annotée comme connecteur, les annotateurs ont précisé quel type de relation de discours était en jeu, en retenant (Section 2.1) les relations de but, de cause ou encore les relations temporelles, avec les étiquettes CONN-But, CONN-Cause et CONN-Narr respectivement. Pour chacune des occurrences de pour introduisant un complément sous-catégorisé, les annotateurs ont précisé si l'élément sous-catégorisant était un verbe, un nom, un adjectif ou un adverbe, avec les étiquettes $C A T-V, C A T-N, C A T-A D J$ et $C A T-A D V$ respectivement. Il a également été demandé aux 
annotateurs de noter les cas où pour introduit une relative sans mot QU avec l'étiquette RelPour et les cas où pour introduit une expression métalinguistique avec l'étiquette META. Au total, les annotateurs devaient choisir entre 9 étiquettes. L'accord inter-annotateurs avant adjudication pour cette première phase est de $82 \%$ avec un kappa à 0,754 , ce qui est honorable pour un jeu de 9 étiquettes.

Dans une seconde phase, les 1162 occurrences de pourVinf du FTB ont été annotées par deux auteurs de cet article avec la participation d'étudiants du Master de Linguistique Informatique de l'Université Paris Diderot (qui avaient lu une version antérieure de cet article). L'accord inter-annotateurs pour cette seconde phase n'a pas pu être mesuré de façon parfaitement rigoureuse mais il est comparable à celui de la première phase. Les résultats de cette seconde phase d'annotation sont donnés dans le tableau ci-dessous.

Que ce soit pour la première ou la seconde phase, des consignes ont été données aux annotateurs pour les participes passés et constructions à verbe support :

- les compléments sous-catégorisés par un participe passé sont annotés $C A T-V$ lorsque le participe passé est employé dans une forme verbale avec un auxiliaire, (48a), sinon ils sont annotés CAT-ADJ, (48b).

(48) a. En fait, l'eurobag est un complément de la ceinture, alors que la version américaine a été conçue pour-CAT-V protéger même sans ceinture. (4063)

b. Mais chacun des deux pays compte aussi sur des projets spécialement conçus pour-CAT-ADJ attirer les investissements étrangers. (3573)

- les compléments sous-catégorisés par un prédicat complexe formé d'un verbe support et d'un nom prédicatif sont annotés $C A T-N$, (49).

(49) Environ cent mille métallurgistes ont déclenché une grève surprise, vendredi 3 juillet, pour-CAT-N protester contre le projet gouvernemental d'instaurer un jour de carence dans le remboursement des arrêts pour maladie. (3226)

\begin{tabular}{|l|ll|}
\hline Connecteur CONN & & 470 \\
- but & 389 & \\
- temporel & 68 & \\
- cause & 13 & \\
\hline Sous-catégorisé CAT & & 621 \\
- verbe & 321 & \\
- nom & 173 & \\
- adjectif & 92 & \\
- adverbe & 35 & \\
\hline Relative sans mot QU RelPour & & 38 \\
\hline Expressions META & & 33 \\
\hline
\end{tabular}

\begin{abstract}
Précisions sur l'accord interannotateurs : le kappa de 0,754 avec un accord de $82 \%$ a été calculé sur les 9 étiquettes. En ce qui concerne l'objectif du FDTB, à savoir distinguer les emplois de pour comme connecteur de ses autres emplois, le kappa avec les seules grandes classes CONN, CAT, RelPour et META est de 0.717 , soit un accord de $85.81 \%$. L'accord à l'intérieur des quatre grandes classes est excellent.
\end{abstract}

La plupart (54\%) des occurrences de pourVinf sont des compléments sous-catégorisés, par un verbe, un nom, un adjectif, ou un adverbe dans l'ordre décroissant de fréquence. On note que les deux étiquettes $C A T-V$ et $C O N N-B u t$ totalisent à elles seules $61 \%$ des cas en étant dans des proportions quasi équivalentes : cette distribution explique la difficulté de l'annotation pour distinguer les occurrences de pour connecteur et pour sous-catégorisée par un verbe (Section 3).

Les divergences entre annotateurs sont dues à quelques erreurs d'inattention et surtout à une centaine d'exemples qui ont nécessité discussion, dont on présente quelques cas typiques ci-dessous.

Les erreurs sont souvent liées au fait qu'il faut prendre en compte le contenu sémantique de l'infinitive. Par exemple en (50a), le nom garanties sous-catégorise l'infinitive en pour, d'où l'étiquette CAT-N. Par contre, 
en (50b), l'infinitive n'est pas complément de garanties, car son contenu n'est pas ce qui est garanti, d'où l'étiquette CONN-But. Autrement dit, la phrase (50a) est paraphrasable par La Russie garantit de soigner Milosevic à Moscou tandis que la dernière phrase de (50b) ne peut être paraphrasée par le canadien garantit d'arracher "sa" grande alliance européenne.

(50) a. La Russie a offert des garanties pour-CAT-N soigner Milosevic à Moscou. (Google)

b. Sept fois plus imposant que son partenaire français, Northern Telecom a réalisé l'an passé un chiffre d'affaires de 8,1 milliards de dollars, soit 42 milliards de francs, et emploie 57000 salariés —, très rentable - il dégage une marge de $6,3 \%$, le canadien a offert un maximum de garanties pour-CONN-But arracher "sa" grande alliance européenne. (2911)

Les désaccords entre annotateurs sont majoritairement des cas où il est difficile de déterminer si la préposition pour est connecteur ou introductrice d'un complément sous-catégorisé par le verbe. En (51), par exemple, il ne vient pas forcément à l'esprit d'un annotateur que l'expression verbale figée frapper à la porte (de) sous-catégorise un complément en pour; pourtant les tests de déplacement et de suppression de la pourVinf dégradent clairement la phrase. La pourVinf a donc été annotée comme sous-catégorisée par l'expression verbale, à l'instar d'un complément qui serait sous-catégorisé par le verbe solliciter.

(51) Pourtant, l'administration a frappé régulièrement à la porte de la Réserve fédérale pour-CAT-V réclamer une nouvelle baisse des taux laquelle s'est finalement produite le 2 juillet. (2768)

L'exemple en (52) a également posé un problème dû à la polysémie du verbe tabler (sur). Dans le sens de compter (sur) qui est celui de (52), ce verbe appelle un complément en pour; dans le sens de s'attendre à, voir L'OFCE table sur une hausse du chômage en Europe, il n'appelle pas pourVinf.

(52) Les gestionnaires tablent ainsi sur la poursuite d'un recentrage de Paribas sur ses métiers de base pour-CAT-V redresser sa situation financière. (3266)

Ajoutons que le modal déontique devoir et le verbe falloir, qui co-occurrent fréquemment avec une pourVinf, (53a-b), ont été l'objet de nombreuses discussions. Le fait que pourVinf peut fonctionner comme cadratif (Charolles, 2005) avec une portée qui s'étend à plusieurs phrases comportant devoir ou falloir reliées par des connecteurs de discours, (53c), nous fait dire que pourVinf n'est sous-catégorisée ni par devoir ni par falloir — contra (Wyler, 2013, p 342) - , pour recevant l'étiquette CONN-But ${ }^{13}$.

(53) a. Résultat : pour conclure certaines affaires, il faut noircir des pages entières. (13944)

b. Luc a dû enlever ses chaussures pour entrer dans le temple.

c. Pour utiliser votre cafetière italienne, il faut remplir d'eau (préférence non calcaire donc minérale) jusqu'à la limite indiquée à hauteur de la soupape dans la partie basse. Ensuite, vous devez remplir le réservoir de café sans le tasser et visser la partie haute. Enfin, il faut laisser chauffer tout doucement en surveillant pour qu'il n'y ait pas d'ébullition ou de réchauffement.

\section{Conclusion et perspectives}

Nous avons identifié quatre classes d'emplois de pour suivie d'une infinitive : connecteur, préposition souscatégorisée, introducteur d'une relative sans mot QU, et introducteur d'une expression méta-discursive. Nous avons de plus raffiné certaines de ces classes en distinguant trois sous-classes d'emplois comme 
connecteur et quatre sous classes d'emplois sous-catégorisés. Un travail d'annotation de 1162 occurrences de pourVinf issues du FTB avec les neuf étiquettes identifiées a révélé un excellent accord inter-annotateurs qui montre que la difficulté majeure est de distinguer entre les emplois de pour comme connecteur et ceux comme préposition sous-catégorisée par un verbe - les distinctions entre les sous-classes de connecteurs ou sous-classes de prépositions sous-catégorisées ne posant guère de problème.

Le double emploi de la préposition pour comme élément sous-catégorisé ou comme connecteur de discours est une chose assez inédite. Aucune autre préposition du français contemporain ne présente cette ambiguïté. A la Section 3, nous avons rapproché ce double emploi des pourVInf de la distinction que l'on fait plus généralement entre les groupes prépositionnels arguments et ajouts. La distinction entre ces deux types de groupes prépositionnels est difficile en syntaxe comme en sémantique, donc au niveau grammatical. La distinction entre les pourVinf arguments et les pourVinf qui traduisent une relation de discours l'est d'autant plus, puisque les deux types de pourVinf relèvent de deux dimensions distinctes de la langue, celle de la grammaire et celle du discours.

A l'issue de ce travail, nous sommes en mesure de faire des propositions afin de compléter les lexiques syntactico-sémantiques du français, par exemple le Lefff (Sagot, 2010), par les nombreux compléments sous-catégorisés par la préposition pour.

\section{Remerciements}

Nous remercions Pascal Amsili, Lucie Barque, Marie Candito et un relecteur anonyme de CMLF pour leur relecture attentive et leurs commentaires fructueux. Nous remercions aussi les étudiants du M2 de Linguistique Informatique (2013-2014) pour leurs efforts d'annotation et leurs rapports d'annotation.

\section{Références bibliographiques}

Abeillé, A., Clément, L. \& Toussenel, F. (2003). Building a treebank for french. In A. Abeillé, Ed., Treebanks. Dordretch: Kluwer Academic Publishers.

Amsili, P. (à paraître). Les ajouts circonstanciels finals. In A. Abeillé \& al., Eds., Grande Grammaire du français. Actes Sud.

Bonami, O. (1999). Les constructions du verbe : le cas des groupes prépositionnels argumentaux. $\mathrm{PhD}$ thesis, Université Paris 7.

Boons, J.-P., Guillet, A. \& Leclère, C. (1976). La structure des phrases simples en français, Constructions intransitives. Genève: Droz.

Cadiot, P. (1990a). Contrôle anaphorique et prépositions. Langages, 97, 8-23.

Cadiot, P. (1990b). A propos du compément circonstanciel de but. Langue française, 86, 51-64.

Charolles, M. (2005). Framing adverbials and their role in discourse cohesion. In Proceedings of SEM-05, p. 194-201, Biarritz, France.

Charolles, M. \& Lamiroy, B. (2012). Les adverbiaux : de la phrase au discours. In Actes du 31e Colloque International sur le Lexique et la Grammaire, Nové Hrady, Czech Republic.

Danlos, L., Antolinos-Bassos, D., Braud, C. \& Roze, C. (2012). Vers le FDTB : French Discourse Tree Bank. In Actes de TALN 2012, Grenoble, France.

Dubois, J. \& Dubois-Charlier, F. (1997). Les Verbes français. Paris: Larousse-Bordas. 
Gross, M. (1975). Méthodes en syntaxe. Paris: Hermann.

Guillet, A. \& Leclère, C. (1992). La structure des phrases simples en français : Les constructions transitives locatives. Genève: Droz.

Huddleston, R. \& Pullum, G. (2002). The Cambridge Grammar of the English Language. Cambridge: Cambridge University Press.

Lamiroy, B. (1983). Les verbes de mouvement en français et en espagnol : étude comparée de leurs infinitives. Amsterdam; Leuven: Lingvisticae Investigationes: supplementa : studies in French \& general linguistics 11, Benjamins : University Press.

Levy, R. \& Andrew, G. (2006). Tregex and Tsurgeon: tools for querying and manipulating tree data structures. In Proceedings of the 5th International Conference on Language Resources and Evaluation (LREC 2006), Giènes, Italie.

Milner, J. (1982). Ordres et raisons de langue. Paris: Le Seuil.

Prasad, R., Dinesh, N., Lee, A., Miltsakaki, E., Robaldo, L., Joshi, A. \& Webber, B. (2008). The Penn Discourse Treebank 2.0. In Proceedings of the 6th International Conference on Language Resources and Evaluation (LREC 2008), Marrackech, Maroc.

Pustejovsky, J. (1995). The generative Lexicon. Cambridge: The MIT Press.

Rossari, C. (à paraître). Les connecteurs. In A. Abeillé \& al., Eds., Grande Grammaire du français. Actes Sud.

Sagot, B. (2010). The lefff, a freely available and large-coverage morphological and syntactic lexicon for french. In Proceedings of LREC 2010, La Valette, Malte.

Tolone, E. (2013). Analyse syntaxique à l'aide des tables du Lexique-Grammaire français. Paris: Etudes universitaires.

Van den Eynde, K. \& Mertens, P. (2003). La valence: l'approche pronominale et son application au lexique verbal. Journal of French Language Studies, 13, 63-104.

Wyler, G. (2013). Manuel de la grammaire française. http://gabrielwyler.com/page000.html.

Zubizareta, M. L. (1985). The relations between morphophonology and morphosyntax: the case of romance causative. Linguistic Inquiry, 16:2, 247-289.

1. Ces ressources sont librement disponibles aux adresses suivantes:

http://infolingu .univ-mlv.fr/DonneesLinguistiques/Lexiques-Grammaires/Telechargement.html

http://pageperso.lif.univ-mrs.fr/ paul.sabatier/Contribution_FondamenTAL.html

http://bach.arts.kuleuven.be/dicovalence/

2. Le terme «cause pragmatique »vient de ce que le contenu de l'infinitive justifie que le locuteur pose la question de la matrice.

3. Pour un exemple anglais comme I have found a box to keep the tapes in (J'ai trouvé une boîte pour stocker les bandes (dedans)), (Huddleston \& Pullum, 2002, p 1176) parlent de relative sans mot WH.

4. Nous laissons de côté les emplois de pour qui s'observent principalement dans la langue parlée, par exemple, l'emploi de pour à la place de de comme complémenteur d'une infinitive extraposée, (i), ou l'emploi avec répétition de la matrice, (ii).

(i) Ça coûte cher de/pour restaurer l'église. (Wyler, 2013)

(ii) Pour être retors, il est retors. (Wyler, 2013)

5. Dans de tels exemples, le remplacement de pour par afin de ou dans le but de donne un résultat déviant. 
6. Voir à ce propos la discussion dans (Cadiot, 1990a, page 62) sur les exemples Jean fuit pour (mourir + éviter sa belle-mère + partir + trouver la tranquillité).

7. Une subordonnée adverbiale antéposée peut jouer le rôle de cadratif (Charolles, 2005). Ainsi, dans le discours (i), la portée sémantique de la subordonnée adverbiale comporte la phrase matrice et la phrase suivante, autrement dit, Luc a fait deux choses pour faire plaisir à Marie.

(i) Pour faire plaisir à Marie, Luc a fait une pizza. Ensuite, il a passé l'aspirateur.

8. On contrastera les exemples suivants :

(i) Luc a offert un bijou à Marie, et ce pendant le dîner.

(ii) *Luc n’a pas offert de bijou à Marie, et ce pendant le dîner.

9. On obtient le même type de contraste lorsqu'on focalise pourVinf au moyen d'une clivée :

(i) Est-ce que c'est pour faire plaisir à Marie que Luc a fait une pizza?

(ii) *Est-ce que c'est pour finir sa thèse que Luc s'est dépêché ?

10. Il peut cependant exister des irrégularités. Ainsi, avec la paire tenter-tentative, le verbe sous-catégorise de, le nom pour.

11. Un artefact a une couleur, ce qui n'est pas le cas d'une astuce.

12. L'exemple (i) a été annoté avec une relative sans mot QU, son antécédent est le nom homme mais on peut considérer qu'il remplace le nom de fonction président.

(i) Chez Carrefour, le président a été remercié du jour au lendemain, les actionnaires voulant un autre homme pour mener une nouvelle stratégie après le temps des acquisitions (2824).

13. Néanmoins, le verbe falloir sous-catégorise une pourVinf exprimant une cause pragmatique dans un exemple comme (i). En effet, la suppression de pourVinf dans un tel exemple débouche sur un résultat déviant.

(i) Il faut être stupide pour croire une chose pareille. 